\title{
VSSAgri: A Vegetation Surveillance System for precision Agriculture application
}

\author{
Amine Saddik ${ }^{*}$, Rachid Latif ${ }^{1}$, Abdelhafid El Ouardi ${ }^{2}$, Mohamed Elhoseney ${ }^{3}$ \\ ${ }^{1}$ Laboratory of Systems Engineering and Information Technology LISTI, National School of Applied Sciences, Ibn Zohr University \\ Agadir, Morocco \\ ${ }^{2}$ SATIE, ENS, Paris Saclay University, Orsay, France \\ ${ }^{3}$ Department of Computer Science,American University in the Emirates, Dubai, UAE \\ *Corresponding email: Amine.saddik@edu.uiz.ac.ma
}

\begin{abstract}
The integration of low-cost systems in precision agriculture applications has shown great benefits, both in terms of the variety of applications and the tools used. In this paper, we present a monitoring platform for agricultural applications based on low-cost systems - also, a review on the different applications of precision agriculture. The proposed platform contains a guidance system based on stepper motors, as well as an embedded architecture for processing the various applications. The guidance system helps to drive the system in the vertical axis for multispectral camera processing. The guidance system helps to move the system in the vertical axis for processing multispectral cameras and in the horizontal axis for the movement of the two bars that will support the system. This platform was inspired by the principle of the scanner, which aims to extract all the information by sliding the scan tool. In the same way, in our case, the sliding will allow us to process the whole agricultural field. The platform proposed in this work has shown that it can be used in different types of the greenhouse, as well as for all types of applications.
\end{abstract}

Keywords. Low-Cost systems, Precision agriculture, VSSAgri plateform, embedded architecture.

\section{Introduction}

Precision agriculture is a recent research field based on the use of sensors and systems to increase production in agricultural fields [1-3]. These systems and sensors can be found on several technologies and a variety of applications. Agriculture generally has three categories. The first focuses on large agricultural fields that contain several thousand hectares of agricultural products. This type of field requires the use of monitoring systems that can cover a large area, such as unmanned aerial vehicles and high-resolution satellite images. These approaches are based on several sensors depending on the chosen application [4-6].

On the other hand, medium-sized agricultural fields require the use of ground robots equipped with autonomous navigation systems. These robots move through the agricultural fields in an autonomous way to extract useful information from the agricultural areas [79]. If we have small agricultural fields or greenhouses, the most optimal solution will be discussed in this work.

The literature in the agricultural field monitoring proposes various solutions to ensure the efficiency and reliability of the results obtained among the proposed works, R.-Delgado et al. 2021, to monitor nitrogen and nitrogen in olive trees in the Spanish region [10]. Also,
J. Lu et al. 2021 proposed an approach for monitoring potassium in rice plants; in this work, the authors were based on a hyperspectral camera and an unmanned aerial vehicle [11]. As well as S. Rabab et al. 2021 proposed a template for the detection of lines in crops based on agricultural fields [12]. In the case of the greenhouse, R. Liu et al. 2021 proposed a system for monitoring temperature and humidity [13]. Z. Huang et al. 2021, offers a robot with centimetric accuracy for greenhouse applications [14]. The authors in [15] have proposed a monitoring system based on an embedded architecture to monitor agricultural fields [15]. Good monitoring in agricultural fields is based on low-cost systems either in terms of the sensors used or the embedded architecture that will handle the different applications. For this reason, we propose a low-cost system for crop monitoring in agricultural fields.

Our work is based on the proposition of an electromechanical system for the monitoring of agricultural fields, more precisely, the greenhouses. Thus, we will discuss the design of the VSSAgri platform, which aims to propose a low-cost system to monitor vegetation in greenhouses. We will also review the different applications that this platform can handle. The design of the VSSAgri system has shown robustness in terms of the number of applications that

* Corresponding author: amine.saddik@edu.uiz.ac.ma 
can be processed, as well as flexibility at the system level. These data encourage us to implement it in a real scenario based on a low-cost system for movement control and an embedded system that will do the processing.

Our paper starts with introduction after that a review and overview based on precision agriculture application. The third part dedicated for our proposed platform for vegetation surveillance and finally a conclusion.

\section{System and application in precision agriculture}

K.P. Ferentinos et al., 2018 present a disease detection technique based on a conventional $\mathrm{CNN}$ neural network. This proposed technique is based on the recognition of plant leaves in order to classify the plant. This paper's evaluation of the proposed algorithm is based on a GPU system type Nvidia GTX1080 with a frequency of $1607 \mathrm{Mhz}$. The programming language used is CUDA, with an accuracy of classification that reaches $99.53 \%$. The paper's authors were based on several models AlexNet, GoogLeNet, VGG, AlexNetOWTBn, Overfeat.

The evaluation of accuracy has shown that the VGG model is more accurate than the other models. The database used in this work contains almost 88,000 images separated into infected sheets and breasts. This database includes 58 classes; each image contains a sheet with their diseases; in the same database, we also have unaffected sheets. The authors have separated this database into two types, one for the validation of the algorithm and the other for the algorithm's training. The first part of the tests contains 17,600 images and the rest for the training. After evaluating this database, they found that an image's processing takes $2 \mathrm{~ms}$, which implies that the real-time constraint is largely respected. But the weak point of this evaluation is the lack of detail on the implementation part, which makes the optimization of the proposed approach difficult. However, the main objective of developing this type of application is the boarding in embedded systems to have autonomous processing [16].

W.Yang et al., 2019 proposed a system for monitoring cold damage in maize fields. The authors used a conventional neural network to extract information from images with visible and near-infrared bands on a range of spectral bands between 450 and 885 $\mathrm{mm}$. After collecting the images, they used filtering techniques such as the Savitzky-Golay filter to eliminate noise when taking the database.

The algorithm proposed in this study is divided into five parts. The first step consisted of collecting the database with a hyperspectral camera type PIKA II, which has a spectral band between 395 and $885 \mathrm{~mm}$, a frequency of $34 \mathrm{~Hz}$, and an integration time of $6.5 \mathrm{~ms}$. The second part is based on plants' sampling; in this part, they divided the plants into 2 parts, one containing control plants and the other cold. The hyperspectral camera used to collect ten images containing 6 plants divided into $3 \times 3$. The third step is the calibration of the collected images to eliminate darkness. The fourth step is to apply the deep learning model to train the database collected. The last step for data processing; this step is based on ENVI version 4.8 and Matlab 7.1. first, it takes the collected data and selects the region of interest, and then it takes 3600 pixels in each image to apply the CNN model. The paper's strong point is the application of deep learning techniques to increase the yield of agricultural fields. Still, no embedded implementation has been processed except for the software used, which implies that the proposed algorithm's portability to a real case in an embedded system cannot be achieved [17].

Y. Pang, et al, 2020 proposed a new MaxArea mask method to segment agricultural fields. The work presents an algorithm that allows the improvisation of crop lines in maize fields based on UAV images. The proposed algorithm was evaluated in a 25-hectare agricultural field with an image resolution of $25 \mathrm{~mm}$. The robustness of the algorithm was tested on a database divided in two different periods of the year with an accuracy of $95.8 \%$. The model proposed in this study was tested on an Nvidia Titan Xp GPU card; this GPU has a frequency of $1583 \mathrm{Mhz}$ and a Cuda core number 384.

The algorithm proposed in this study is divided into 4 steps; the first one consists of collecting the database based on a UAV with a time of flight of $15 \mathrm{~min}$. The second part is based on the division of fields with several data depending on the UAV flight alteration, in order to have several patches of agricultural fields. The second step is based on the processing of each image by applying the RCNN model based on the MaxArea mask, then calculating the rotation angle. The last part focuses on applying the algorithm, which will detect each line of maize for counting. Figure 1 shows the algorithm used [18].

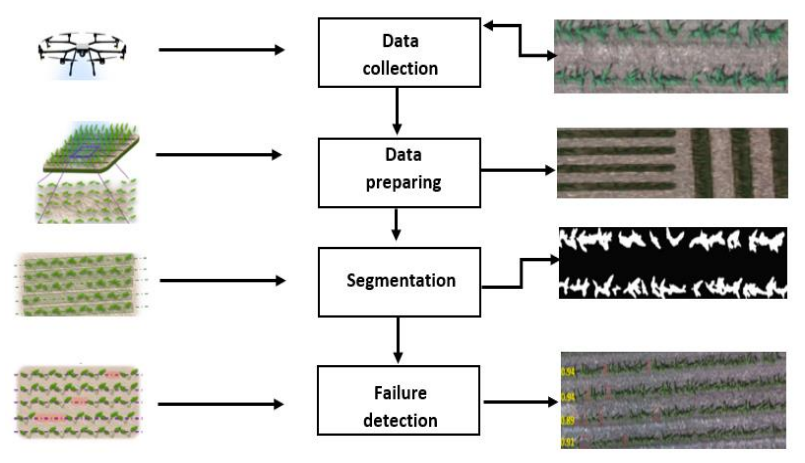

Fig. 1. Algorithm Overview.

The highlight of the paper is the proposal of a new MAxArea mask technique for the monitoring of agricultural maize fields, as well as the increase in yield. But the GPU system used is known for its high energy consumption, which implies a short flight time, and no optimization or processing time study has been proposed for real-time processing.

A.Casado-Garcíaa et al. 2020 present in their works a technique based on the YOLO model for the 
monitoring of stomata in plants in order to determine the gas exchange and the quantity of water. After evaluating the algorithm, the results showed an F1 score of 0.91 for two sets of images. The interpretation of the results is made through software developed by the authors (LabelStoma) to facilitate the use of the chosen model. The authors' work is divided into two parts: the biological part and the other for the calculation part. In the biological part, they used images of different species collected in 3 different laboratories.

The plants used in this work have been submitted on several environmental conditions. Generally, the data used are divided into a common bean, barley, and sobean with image resolution $1280 \times 960,1728 \times 1296$, $1600 \times 1200$ respectively. After the database construction, they used an Nvidia RTX 2080 TI GPU card with a frequency of $1545 \mathrm{MHz}$ and Turing architecture for model training with $86 \%$ of the images and the rest for testing. The paper showed that deep learning techniques could be used without manual diagnostics constraints, and the model used also showed great efficiency. However, the paper was based on only one model, limiting the performance of using this technique on several models to make a comparative study [19].

Within the context of the monitoring and management of fruit leaves, Philipe A. Dias et al. 2018 have proposed segmentation on convolutional neural networks to increase detection precision compared to other traditional works based on artificial intelligence that suffers from an environmental problem. However, these techniques require specific conditions to perform different algorithms based on artificial intelligence.

The authors have proposed a robust method for detecting various types of flowers and have improved $\mathrm{CNN}$ to have a high detection sensitivity based on the refinement of several apple flower images. The algorithm used in this work is based only on the processing without pre-treatment or specific training, and the flowers used are pear, peach, and apple.

The database used in this paper is divided into 4 collections with two cameras, a GoPro HERO5 with a resolution of $2704 \times 1520$. the GoPro Hero5 camera has a speed between 30 and 240 fps depending on the resolution used. The second camera used is a Canon EOS $60 D$ with a $5184 \times 3456$ resolution; this camera was used to collect the apple flowers. On the other hand, the GoPro camera for peach and Pear. Figure 2 shows the tools used and the images obtained [20].
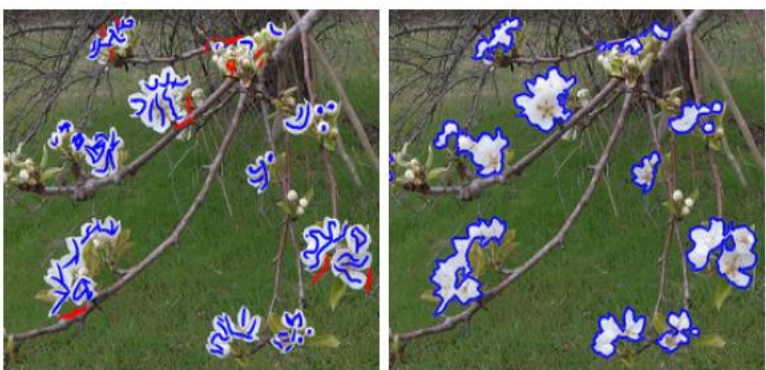

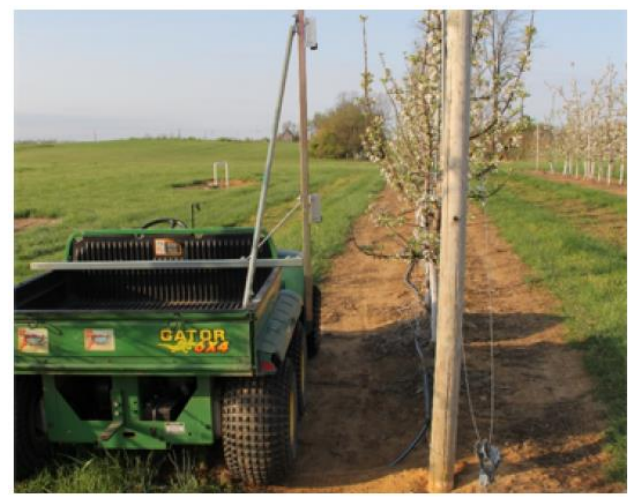

Fig. 2. System tools Overview [20].

The algorithm's evaluation was based on an Intel Xeon E5-2620 CPU system with a frequency of $2.4 \mathrm{Ghz}$ and a P600 GPU card. The time study showed that the system takes $50 \mathrm{~s}$ for the evaluation of each image and $5 \mathrm{~s}$ to record the desired portrait. The strong point of the paper is the proposal of an improved CNN technique for the detection of flowers of different fruits and a study of the temporal constraint presented. However, the paper's weak point is accuracy; the authors did not present an accuracy study with the literature's different papers.

The increase of production in agricultural fields is generally linked to the quality of the monitoring tools and the quality of the fruit. However, different fruit types are very sensitive to various damages and therefore need permanent monitoring and follow-up to have good quality production and yield. The work of X.wang et al. 2020 was based on a fully convolutional network (FCN) to segment the apple flowers. The proposed technique's evaluation has shown an F1 score of $85.6 \%$ with a variation in different. This variation showed that the algorithm used gave strong and accurate results. Figure 3 shows the system used [21].

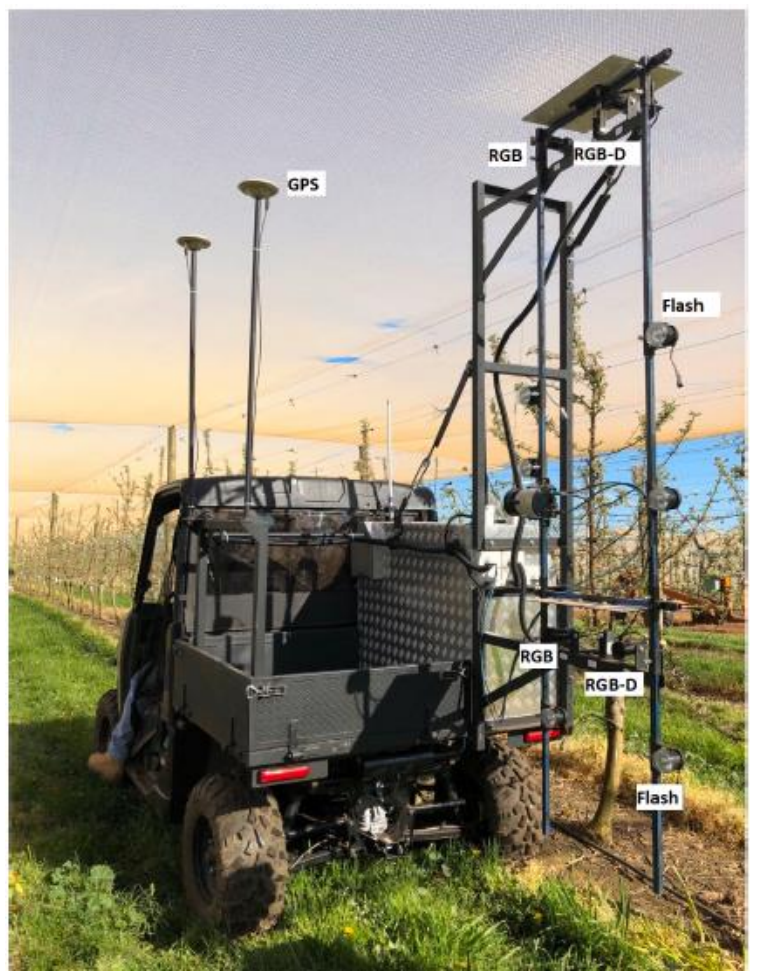

Fig. 3. Proposed system based robot [21]. 
The figure shows that the authors used a car with a GPS RTK, RGB, and RGBD camera. The use of this type of tool reflects that the vehicle gives images on very precise coordinates. The system used in this work is based on a GPU card type Nvidia 1050 and a CPU intel core I7. The time evaluation shows that the proposed algorithm takes about 40 minutes for data input on the proposed system; the chosen resolution is $320 \times 640$. The processing time has been presented for $0.12 \mathrm{~s}$ in $480 \times 800$ resolution and 0.32 for $640 * 1280$. Besides, the strong paper bridge is the proposal of an autonomous system based on the image precision to make the apple leaves' segmentation. On the other hand, the processing time problem is summarized on the real-time constraint on the camera's time-lapse; for example, a frequency of 30 frames/s cannot be processed.

The work of Y. LI et al. 2019 tried to build a model that would merge the density estimation and convolutional neural network $(\mathrm{CNN})$ based approaches to estimate precisely the seed count from an isolated soybean pod image from a unique viewpoint. The proposed technique is based on a new large-scale seed counting database known as the Soybean-pod. The evaluated database contains 500 labeled pod frames with a total of 32,126 seeds, which is the most extensive annotated data set for soybean counts to date. At the same time, they also used the annotation data to simultaneously produce a field truth density map by convolving a Gaussian kernel. A simple but powerful method was developed to allow the pod images to be elucidated into a seed density map with a two-column CNN (TCNN) and, therefore, perform the seed count in the end. The evaluation of the error showed that the root means square error a 13.62. The system used in this work is based on a 1080 Ti GPU card with a CPU E5-2630 based on 20 processors and a frequency of $2.2 \mathrm{GHz}$. The work's main point is using a two-column CNN model that increases the accuracy and reduces the error. However, the work has not been compared with other states of the art techniques [22].

We can conclude that most of the systems mentioned are based on desktop or laptop. This system is characterized by the high frequency of CPUs and GPUs, which reduces the processing time. On the other hand, embedded systems suffer from a frequency problem. This problem is due to the use of minimized architecture, which limits the frequency of the systems. However, the implementation of treatment and monitoring algorithms in agricultural fields for several applications in conventional and solid machines such as desktop or laptop does not imply importing these algorithms in dedicated architectures. For this reason, a thorough study of different algorithmic and architectural characteristics is necessary for the adaptation of these systems for real cases. In agricultural fields, it is always necessary to take into account the different energy and memory constraints in order to have a robust and solid system for making precise calculations.

\section{Proposed platform and discussion}

\subsection{Greenhouse type}

Greenhouses offer a robust solution for increasing the yield of agricultural products. The main role of the closed greenhouse is to protect the crops from different environmental changes and different types of diseases. As well as this greenhouse is used to create the perfect environment for the plants to give the maximum production. The construction of the greenhouse is a very special one, consisting of a translucent glass roof to let the necessary light through for the plants. A climate manager is very important in creating the ideal environment for the cultivation of the crops and a temperature and area manager to control the temperature and environment of the plants. In general, greenhouses can be divided into four categories: greenhouses in agricultural fields, underground greenhouses, photovoltaic agricultural greenhouses, and greenhouses integrated with building roofs [23]. Figure 4 shows the different types of closed greenhouses, with A quonset, B tri-penta, C dome, D gothic arch, E slant-leg, F a-frame, F half-pit, and $\mathrm{G}$ gable roof.
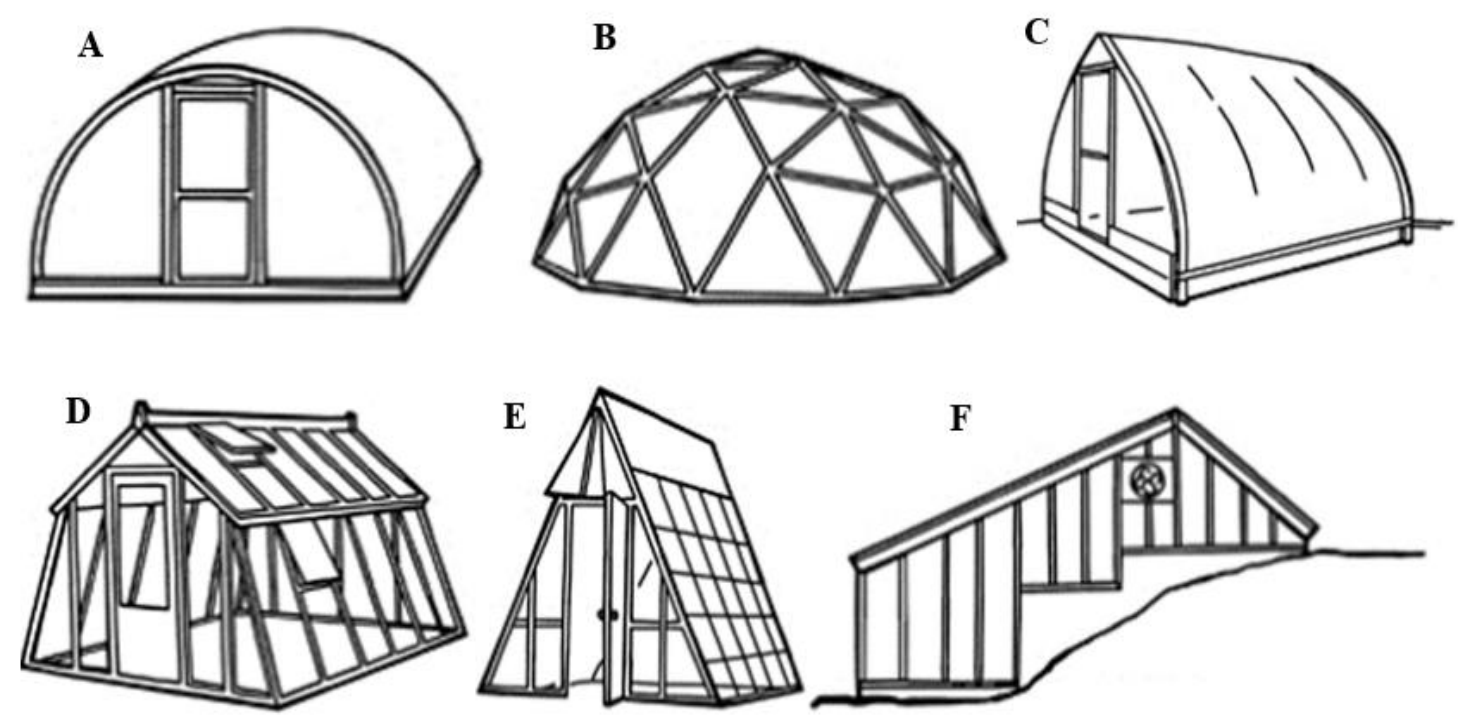

Fig. 3. Closed greenhouses types. 


\subsection{VSSAgri Platform}

Our system consists of proposing an autonomous prototype for the surveillance of Quonset greenhouses. It consists of a multispectral camera type parrot sequoia + and an embedded architecture type CPU-GPU. The board chosen in our case is dedicated to the monitoring of vital indices such as NDVI and NDWI. According to the evaluation proposed in [15], this architecture can process up to 20 frames/s, which is sufficient for real-time processing. Also, the multispectral camera timelapse is around 2 frames/s for the different band types. The system takes into consideration two displacements, the first one between the two ends of the used bars and the second one along the length of the closed greenhouse. Figure 5 shows the monitoring system.

The system contains two bars at the end of the platform; these two bars are attached to sliding support. Image A shows the system in state mode; the moving box contains the onboard card that will do the surveillance of the indices as well as a power source for the system's power supply. The parrot sequoia + camera is used to extract different images with different bands. Image B shows the box moving on a sliding cable, allowing the box to move from right to left. This movement is controlled by a stepper motor which is used to follow the timing laps. The configuration of this motor depends on the time of the image. The reason why we used the stepper motor and not another one is to keep the box movement synchronized with the images taken in the camera.

Image $\mathrm{C}$ shows the complete displacement of the box to the left, which means that the system has finished processing. After that, the two bars are moved using a motor based on the number of pixels taken from the camera and the distance in each pixel to calculate the correct displacement of the two bars. Image $\mathrm{D}$ shows the movement of these two bars to ensure that the whole greenhouse is processed. Picture E shows that the two bars have been moved green in the middle.

The movement of the two motors is calculated beforehand. It depends on the height of the barrier and the type of cameras used to ensure that the entire greenhouse is monitored. This closed greenhouse will contain the sensitive plants as well as the large surface of the closed greenhouse. Therefore, the VSSAgri platform is dedicated to greenhouses with a large surface area.
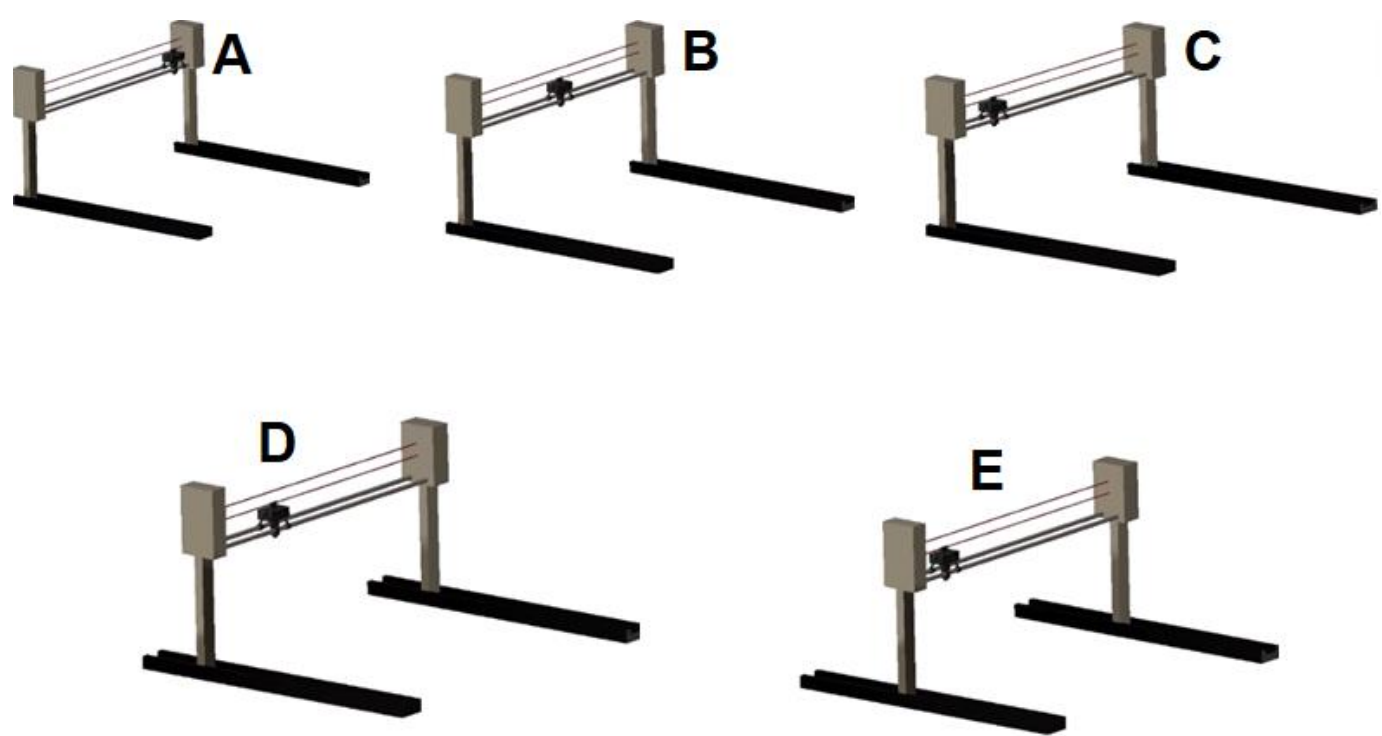

Fig. 5. Proposed functionality system [23].

The advantage of the system is its low cost and simplicity of integration into any type of closed greenhouse. This solution can also be used for index monitoring, plant counting and disease detection, the only thing that will change is the type of camera.

This is because each application requires a dedicated camera; for example, counting or detecting diseases and weeds requires an RGB camera. On the other hand, the monitoring of indices is based on multispectral cameras. In the VSSAgri Platform, we have used the multispectral camera as it gives RGB images and images with different bands separated, which makes the system dedicated to any kind of application. The greenhouse targeted in our study is the Quonset type. Figure 6 shows the system that monitors the desired plants.

After the system finishes the processing, a central analysis unit is installed to interpret the results and react to 
the different plants. This reaction is dependent on the type of plantation that exists in the closed greenhouse.

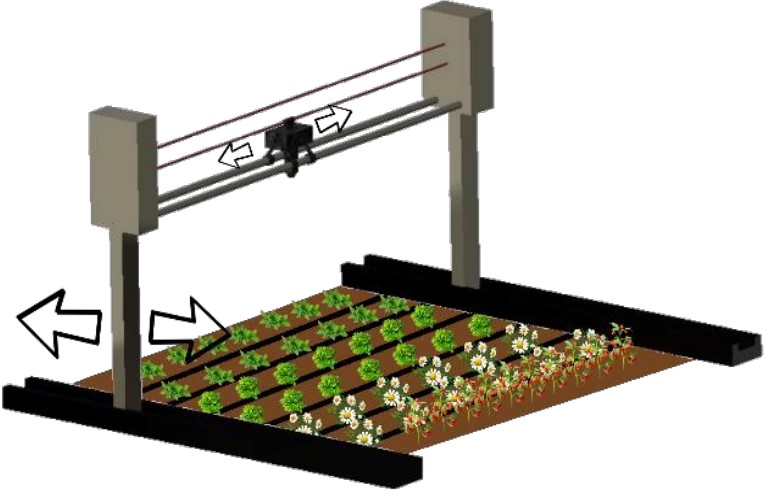

Fig. 6. VSSAgri overview.

In our system, we have two types of movement, one is for scanning the plants in a horizontal direction, and the other is for vertical movement. These two movements, leftright, and right-left will ensure that all plants in the desired greenhouse are processed. In figure 6 , we have varied the plants to show that the system can monitor all plants.

\subsection{Discussion and Future work}

The VSSAgri platform is inspired by the principle of a scanner that slides over the whole sheet to extract useful information. In the same way, the proposed system is dedicated to doing the same task, except that the support used here is also moving. As a result, the VSSAgri platform presents a low-cost solution compared to the different solutions proposed in unmanned aerial vehicles based on expensive tools. In our system, we have an embedded board that contains a CPU board and another GPU for the acceleration of the processing algorithms. A raspberry board is used to control the motors for the different movements. A ventilation system is also used to ensure the correct operation of the boards in the case used. Figure 7 shows the system integrated into a closed greenhouse.



Fig. 7. Closed greenhouse based VSSAgri platform.

Figure 7 shows our VSSAgri platform installed in the closed greenhouse containing the different crops. In work [15], the authors showed that the used system based on the XU4 board could process up to 20 frames/s, as well as the work of [24] presented a very low time compared to [15]. Still, the embedded system used Jetson TX1 consumes a lot of energy compared to the
XU4 board, making the energy consumption a very important parameter as the system is based on a battery that excludes the use of energy-consuming architecture.

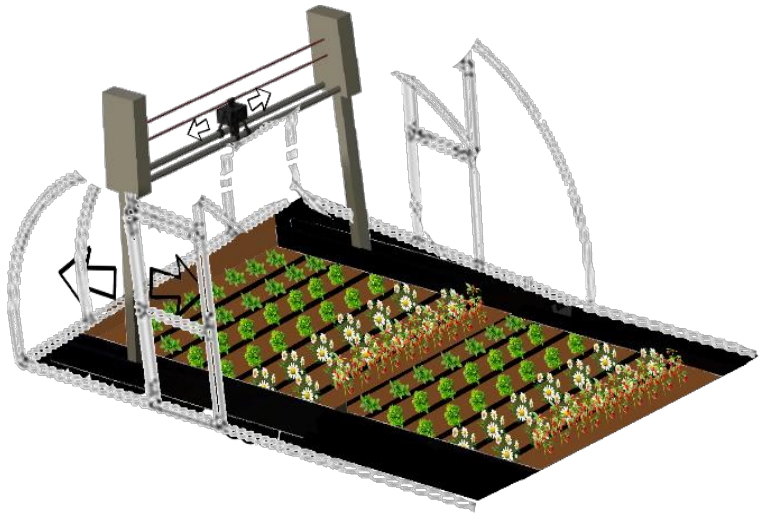

Fig. 8. Larger greenhouse based platform[23].

The system proposed in this paper is suitable for all types of greenhouses, which makes it a general system for all uses. Figure 8 shows the use of the system in a larger greenhouse than the one proposed in figure 7 .

As future work, we aim to achieve real implementation in a greenhouse. As well as using the Hardware In Loop to validate the platform based on a real reference, we also aim to integrate several other applications to ensure that the system will process the maximum possible number of applications. We also aim to improve the VSSAgri platform in order to design a robust, reliable, and real-time system.

\section{Conclusion}

In this work, we study the different applications proposed in the literature for precision agriculture applications. We also discuss the feasibility of a platform for the autonomous monitoring of the various applications in the greenhouse. The VSSAgri platform proposed in this work aims to present a robust system for monitoring the different crops to increase the yield of the agricultural fields. The system contains two parts, the first one for the displacement of the box that carries the processing architecture, including the embedded system used as well as the camera. The second part contains two barriers considered as a support for the platform's displacement to guarantee the monitoring of the totality of the targeted greenhouse. The platform proposed in this work has been based on the low-cost aspect to decrease the cost of tracking in the agricultural fields to guarantee the maximum yield with the least of the tools

\section{Acknowledgement}

We owe a debt of gratitude to the National Centre for Scientific and Technical Research of Morocco (CNRST) for their financial support and for their supervision (grant number: 19 UIZ2020). 


\section{References}

1. R. LATIF, A. SADDIK and A. ELOUARDI, "Evaluation of Agricultural Precision Algorithms on UAV Images," 2019 International Conference of Computer Science and Renewable Energies (ICCSRE), 2019, pp. 1-4, doi: 10.1109/ICCSRE.2019.8807604.

2. Sishodia, R.P.; Ray, R.L.; Singh, S.K. Applications of Remote Sensing in Precision Agriculture: A Review. Remote Sens. 2020, 12, 3136. https://doi.org/10.3390/rs12193136.

3. Kolady, D.E., Van der Sluis, E., Uddin, M.M. et al. Determinants of adoption and adoption intensity of precision agriculture technologies: evidence from South Dakota. Precision Agric 22, 689-710 (2021). https://doi.org/10.1007/s11119-020-09750-2.

4. Zhang, Q., Chen, Q., Xu, Z. et al. Evaluating the navigation performance of multi-information integration based on low-end inertial sensors for precision agriculture. Precision Agric 22, 627-646 (2021). https://doi.org/10.1007/s11119-020-09747$\mathrm{X}$

5. Afrasiabian, Y., Noory, H., Mokhtari, A. et al. Effects of spatial, temporal, and spectral resolutions on the estimation of wheat and barley leaf area index using multi- and hyper-spectral data (case study: Karaj, Iran). Precision Agric 22, 660-688 (2021). https://doi.org/10.1007/s11119-020-097499

6. Kazama, E.H., da Silva, R.P., Tavares, T.O. et al. Methodology for selective coffee harvesting in management zones of yield and maturation. Precision Agric 22, 711-733 (2021). https://doi.org/10.1007/s11119-020-09751-1

7. Latif R., Jamad L., Saddik A. (2021) Implementation of Hybrid Algorithm for the UAV Images Preprocessing Based on Embedded Heterogeneous System: The Case of Precision Agriculture. In: Hassanien A.E., Darwish A., Abd El-Kader S.M., Alboaneen D.A. (eds) Enabling Machine Learning Applications in Data Science. Algorithms for Intelligent Systems. Springer, Singapore. https://doi.org/10.1007/978-981-336129-4 11

8. Stefan K. Ericson, Björn S. Åstrand, Analysis of two visual odometry systems for use in an agricultural field environment, Biosystems Engineering, Volume 166, 2018, Pages 116-125, https://doi.org/10.1016/j.biosystemseng.2017.11.0 09.

9. R. Latif and A. Saddik, "SLAM algorithms implementation in a UAV, based on a heterogeneous system: A survey," 2019 4th World Conference on Complex Systems (WCCS), 2019, pp. 1-6, doi: 10.1109/IcoCS.2019.8930783.

10. Rubio-Delgado, J., Pérez, C.J. \& Vega-Rodríguez, M.A. Predicting leaf nitrogen content in olive trees using hyperspectral data for precision agriculture. Precision Agric 22, 1-21 (2021). https://doi.org/10.1007/s11119-020-09727-1
11. Lu, J., Li, W., Yu, M. et al. Estimation of rice plant potassium accumulation based on non-negative matrix factorization using hyperspectral reflectance. Precision Agric 22, 51-74 (2021). https://doi.org/10.1007/s11119-020-09729-z

12. Rabab, S., Badenhorst, P., Chen, YP.P. et al. A template-free machine vision-based crop row detection algorithm. Precision Agric 22, 124-153 (2021). https://doi.org/10.1007/s11119-020-097324 .

13. R. Liu, M. Li, J.L. Guzmán, F. Rodríguez, A fast and practical one-dimensional transient model for greenhouse temperature and humidity, Computers and Electronics in Agriculture, Volume 186, 2021, 106186 , https://doi.org/10.1016/j.compag.2021.106186.

14. Zichen Huang, Tomoo Shiigi, Lok Wai Jacky Tsay, Hiroaki Nakanishi, Tetsuhito Suzuki, Yuichi Ogawa, Kondo Naoshi, A sound-based positioning system with centimeter accuracy for mobile robots in a greenhouse using frequency shift compensation, Computers and Electronics in Agriculture, Volume 187, 2021, 106235, https://doi.org/10.1016/j.compag.2021.106235.

15. Amine Saddik, Rachid Latif, Mohamed Elhoseny, Abdelhafid El Ouardi, Real-time evaluation of different indexes in precision agriculture using a heterogeneous embedded system, Sustainable Computing: Informatics and Systems, Volume 30, 2021, 100506 , https://doi.org/10.1016/j.suscom.2020.100506.

16. Konstantinos P. Ferentinos, Deep learning models for plant disease detection and diagnosis, Computers and Electronics in Agriculture, Volume 145, 2018, Pages 311-318, https://doi.org/10.1016/j.compag.2018.01.009.

17. W. Yang, C. Yang, Z. Hao, C. Xie and M. Li, "Diagnosis of Plant Cold Damage Based on Hyperspectral Imaging and Convolutional Neural Network," in IEEE Access, vol. 7, pp. 118239118248, 2019, doi: 10.1109/ACCESS.2019.2936892.

18. Yan Pang, Yeyin Shi, Shancheng Gao, Feng Jiang, Arun-Narenthiran Veeranampalayam-Sivakumar, Laura Thompson, Joe Luck, Chao Liu, Improved crop row detection with deep neural network for early-season maize stand count in UAV imagery, Computers and Electronics in Agriculture, Volume 178, 2020, 105766, https://doi.org/10.1016/j.compag.2020.105766.

19. Angela Casado-García, Arantza del-Canto, Alvaro Sanz-Saez, Usue Pérez-López, Amaia BilbaoKareaga, Felix B. Fritschi, Jon Miranda-Apodaca, Alberto Muñoz-Rueda, Anna Sillero-Martínez, Ander Yoldi-Achalandabaso, Maite Lacuesta, Jónathan Heras, LabelStoma: A tool for stomata detection based on the YOLO algorithm, Computers and Electronics in Agriculture, Volume 178, 2020, 105751, https://doi.org/10.1016/j.compag.2020.105751. 
20. P. A. Dias, A. Tabb and H. Medeiros, "Multispecies Fruit Flower Detection Using a Refined Semantic Segmentation Network," in IEEE Robotics and Automation Letters, vol. 3, no. 4, pp. 3003-3010, Oct. 2018, doi: 10.1109/LRA.2018.2849498.

21. Xu (Annie) Wang, Julie Tang, Mark Whitty, Sideview apple flower mapping using edge-based fully convolutional networks for variable rate chemical thinning, Computers and Electronics in Agriculture,Volume 178,2020,105673, https://doi.org/10.1016/j.compag.2020.105673.

22. Y. Li et al., "Soybean Seed Counting Based on Pod Image Using Two-Column Convolution Neural Network," in IEEE Access, vol. 7, pp. 6417764185, 2019, doi: 10.1109/ACCESS.2019.2916931.

23. Ahmed Al Makky, A. Alaswad, Desmond. Gibson, A.G. Olabi, Prediction of the gas emission from porous media with the concern of energy and environment, Renewable and Sustainable Energy Reviews, Volume 68, Part 2, 2017, Pages 11441156, https://doi.org/10.1016/j.rser.2016.08.001. 